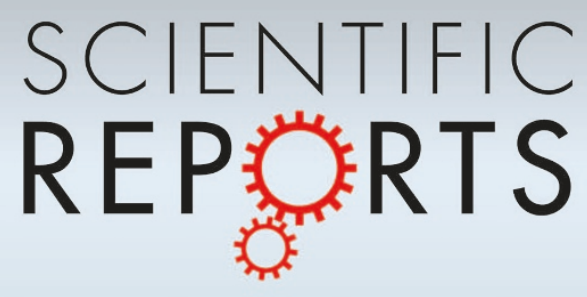

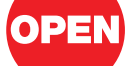

SUBJECT AREAS:

ELECTRONIC STRUCTURE

OF ATOMS AND

MOLECULES

ATOMIC AND MOLECULAR INTERACTIONS WITH

PHOTONS

ULTRAFAST LASERS

ULTRAFAST PHOTONICS

Received

18 October 2012

Accepted

19 December 2012

Published

22 January 2013

Correspondence and requests for materials should be addressed to Z.C. (Zenghu.Chang@ ucf.edu)

\section{Sub-cycle Oscillations in Virtual States Brought to Light}

\author{
Michael Chini' ${ }^{1}$ Xiaowei Wang' ${ }^{1,2}$, Yan Cheng' ${ }^{1}$ Yi Wu', Di Zhao ${ }^{3,4}$, Dmitry A. Telnov ${ }^{3,5}$, Shih-I Chu ${ }^{3,6}$ \\ \& Zenghu Chang'
}

${ }^{1}$ CREOL and Department of Physics, University of Central Florida, Orlando, FL 32816, USA, ${ }^{2}$ Department of Physics, National University of Defense Technology, Changsha, Hunan 410073, China, ${ }^{3}$ Department of Chemistry, University of Kansas, Lawrence, KS 66045, USA, ${ }^{4}$ Department of Applied Physics, Xi'an Jiaotong University, Xi'an, Shanxi 710049, China, ${ }^{5}$ Department of Physics, St. Petersburg State University, St. Petersburg 198504, Russia, ${ }^{6}$ Center for Quantum Science and Engineering, National Taiwan University, Taipei, 10617, Taiwan.

Understanding and controlling the dynamic evolution of electrons in matter is among the most fundamental goals of attosecond science. While the most exotic behaviors can be found in complex systems, fast electron dynamics can be studied at the fundamental level in atomic systems, using moderately intense $\left(\lesssim 10^{13} \mathrm{~W} / \mathrm{cm}^{2}\right)$ lasers to control the electronic structure in proof-of-principle experiments. Here, we probe the transient changes in the absorption of an isolated attosecond extreme ultraviolet (XUV) pulse by helium atoms in the presence of a delayed, few-cycle near infrared (NIR) laser pulse, which uncovers absorption structures corresponding to laser-induced "virtual" intermediate states in the two-color two-photon $(\mathrm{XUV}+\mathrm{NIR})$ and three-photon (XUV+NIR+NIR) absorption process. These previously unobserved absorption structures are modulated on half-cycle $(\sim 1.3 f s)$ and quarter-cycle $(\sim 0.6 f s)$ timescales, resulting from quantum optical interference in the laser-driven atom.

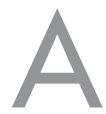

mong the most fundamental optical properties of a material is its absorption of light. Spectrally-resolved absorption has long been used to study atomic structure ${ }^{1}$ and provides a "fingerprint" for identifying atomic and molecular species. With the addition of a moderately intense laser field, the absorption spectrum can be modified, allowing measurement of and demonstrating control over the electron dynamics in the atom on ultrafast timescales. Laser-dressed absorption measurements have recently demonstrated controllable EIT-like phenomena in atoms at extreme ultraviolet $(\mathrm{XUV})^{2}$ and $\mathrm{x}-\mathrm{ray}^{3}$ wavelengths. More recently, transient changes in the absorption of an isolated attosecond pulse in the presence of a synchronized few-cycle laser pulse were observed in valence electron wavepackets in field-ionized krypton ${ }^{4}$, autoionizing states of argon ${ }^{5}$ and Stark-shifted excited states of helium ${ }^{6}$, allowing measurement of electron dynamics on the few-femtosecond and even sub-laser-cycle timescale. Here, we study the absorption of an isolated attosecond pulse in the vicinity of the helium absorption line manifold corresponding to excitation of an electron from the ground state $\left(1 \mathrm{~s}^{2}\right)$ to the $1 \mathrm{~s} n$ p excited states. By adding a moderately intense $\left(\sim 10^{13} \mathrm{~W} / \mathrm{cm}^{2}\right)$ near infrared (NIR) few-cycle laser field, new absorption features appear that correspond to laser-induced "virtual" intermediate states in the two-color multiphoton excitation of 1 sns and $1 s n d$ states, for which direct dipole transitions from the ground state through the absorption of XUV light are forbidden. By changing the delay between the attosecond pulse and the NIR laser field, the absorption strengths of the virtual state absorption features are modulated on the attosecond timescale. These modulations highlight the mechanisms behind the time-dependent formation of spectrally narrow absorption features, as they result from spectral interference in the dipole emission of the laser-driven atom. The strong bound-bound and bound-continuum state couplings enabled by the strong laser are further revealed through energy level shifts, such as Autler-Townes splitting as well as strong AC ("alternating current", as in the oscillating electric field of the laser) Stark and ponderomotive shifts of the 1snp states which evolve on the sub-cycle timescale.

\section{Results}

The experiment (Fig. 1a, Methods) was performed using the attosecond transient absorption technique ${ }^{4-6}$, in which the absorption of an isolated attosecond pulse is measured in the presence of a delayed laser field. The experimental setup combines the high temporal resolution afforded by the isolated attosecond probe pulse in an actively stabilized interferometer ${ }^{7}$ with the high spectral resolution of XUV absorption spectroscopy ${ }^{8}$. Compared 


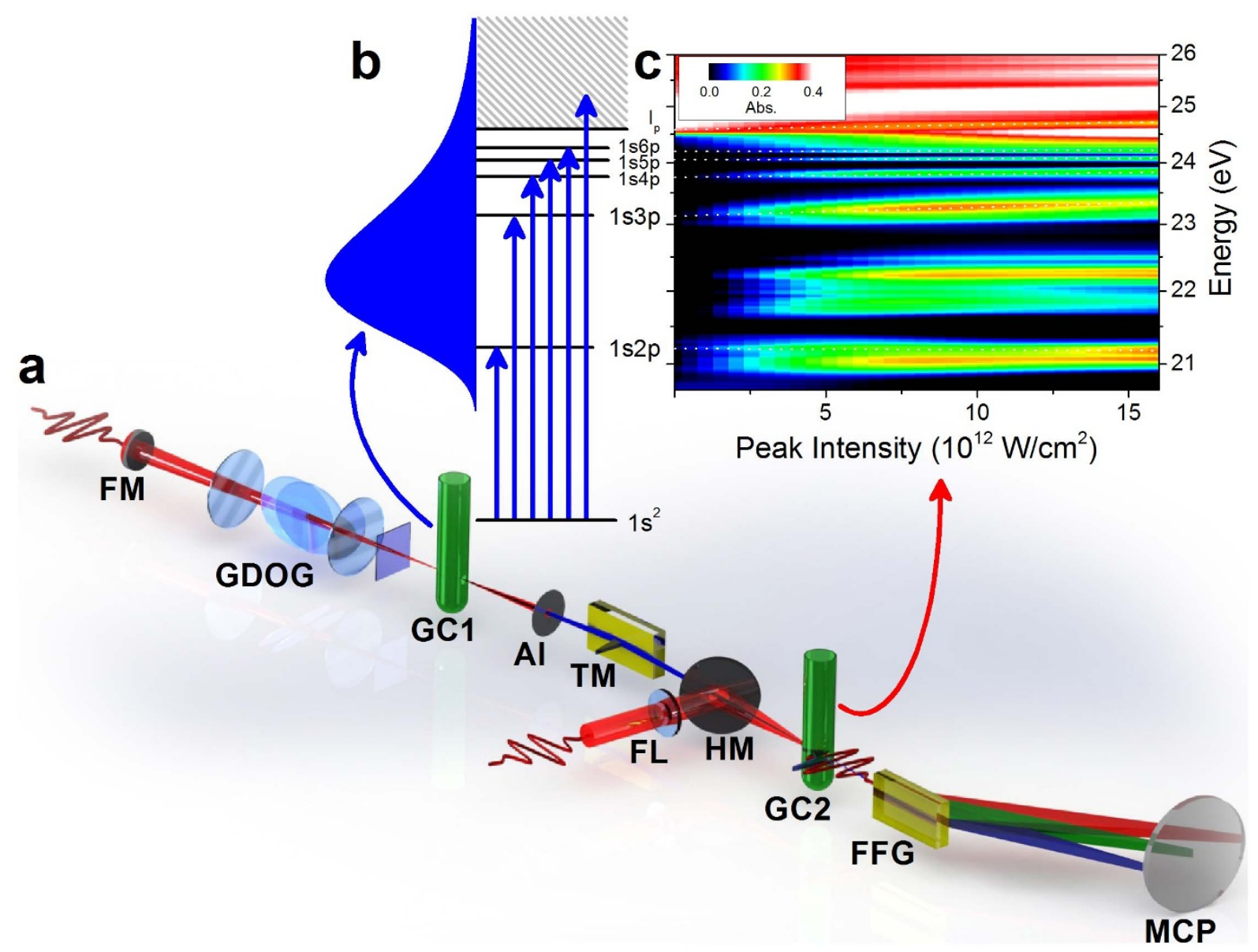

Figure $1 \mid$ Laser-dressed attosecond XUV absorption in helium. (a) Schematic of experimental setup for attosecond transient absorption. FM: focusing mirror, GDOG: generalized double optical gating optics (see Methods), GC1: argon or xenon-backed gas cell for attosecond pulse generation, Al: aluminum foil filter, TM: toroidal mirror, FL: focusing lens, HM: hole-drilled recombination mirror, GC2: helium-backed gas cell for absorption measurements, FFG: flat-field XUV grating, MCP: microchannel plate and phosphor screen detector. (b) Helium 1snp energy level diagram showing schematic XUV spectrum and states accessible for excitation by the attosecond pulse. (c) Measured absorbance spectrum as a function of the dressing laser intensity when the attosecond XUV pulse overlaps in time with the peak of the NIR laser pulse. The dotted lines trace the Stark-shifted absorption lines. At an intensity of $\sim 2.5 \times 10^{12} \mathrm{~W} / \mathrm{cm}^{2}$, several new absorption features appear in the vicinity of the $1 \mathrm{~s} 2 \mathrm{p}$ state, which dynamically evolve as the laser intensity is increased.

to attosecond photoelectron spectroscopy ${ }^{9}$, the attosecond transient absorption technique allows direct and simultaneous observation of the excited state dynamics and quantum interferences both above and below the ionization potential. Bound and continuum state dynamics can therefore be measured with high fidelity in low-intensity laser fields, where the probability of ionization (and therefore the photoelectron yield) is small, as well as in high-intensity laser fields where the contribution of multiple ionization channels complicates the interpretation of photoelectron spectra.

Fig. $1 \mathrm{~b}$ shows the energy level diagram of the helium $1 \mathrm{~s} n \mathrm{p}$ excited states $^{10}$, which extend from $21 \mathrm{eV}$ above the ground state to the first ionization potential at $24.58 \mathrm{eV}$, along with the absorbance measured as a function of the dressing laser intensity at a fixed time delay where the peak of the NIR laser pulse overlaps with the attosecond pulse. The broad, continuous spectrum of the isolated attosecond pulse allows us to observe the absorption lines corresponding to each individual state as well as the absorption above the ionization potential simultaneously. At the lowest dressing laser intensities, the absorption features corresponding to the 1snp excited state manifold can hardly be seen, since these states have long ( nanosecond) lifetimes corresponding to absorption line widths below $1 \mu \mathrm{eV}^{11}$. Such narrow features cannot be observed with the XUV spectrometer, which has a resolution of $\sim 50 \mathrm{meV}$. As the intensity of the dressing laser is increased, however, the lifetimes of the excited states are reduced, resulting in relatively broadened absorption lines. More interestingly, the absorption in the vicinity of the 1 s $n$ p excited state manifold is changed significantly with the addition of the strong laser field $^{12-14}$. For the $1 \mathrm{~s} 3 \mathrm{p}(23.09 \mathrm{eV})$ and higher-lying states (leading to the absorption edge at the ionization potential), these changes amount to energy shifts of the absorption lines, which correspond to AC Stark ${ }^{6}$ and ponderomotive shifts. However the laser-dressed $1 s 2 p$ absorption line, which was not observed in previous experiments, exhibits more complicated structure, including energy level splitting and the formation of new absorption sub-structures (near $22 \mathrm{eV}$, becoming prominent at intensities above $\sim 2 \times 10^{12} \mathrm{~W} / \mathrm{cm}^{2}$ ) in addition to a relatively small AC Stark shift.

The observed absorption sub-structures arise from transient virtual states of the laser-dressed atom, which can be understood in terms of two-color two-photon (XUV+NIR) and three photon (XUV+NIR+NIR) absorption processes leading to dipole-forbidden final states after interaction with both light fields. Each substructure therefore represents a virtual intermediate state in the excitation of a given $1 \mathrm{~s} n l$ excited state through absorption of an XUV photon and one or more NIR photons, as shown schematically in Fig. 2a. These transient states are analogous to Floquet sidebands of the laser-dressed atom, and exist only when both the XUV and NIR lasers interact with the atom. We therefore classify each virtual state absorption feature by its corresponding final state and the 

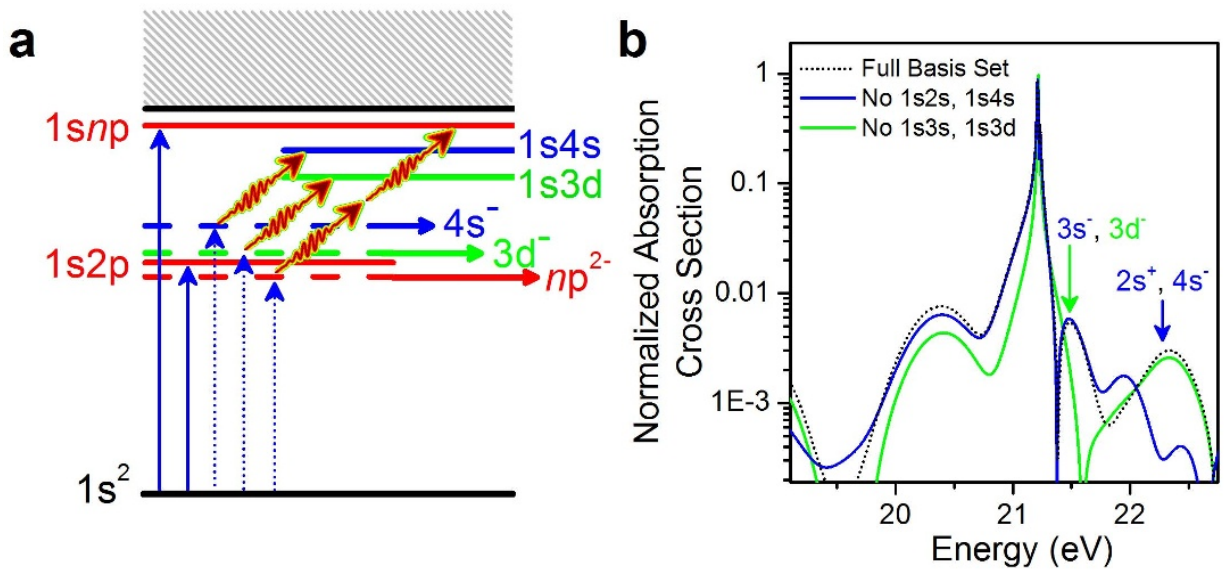

Figure 2 Excitation of transient virtual excited states through two-color multi-photon absorption. (a) Schematic diagram of two-color two- and threephoton absorption pathways to the $1 \mathrm{~s} 3 \mathrm{~d}$ (green), $1 \mathrm{~s} 4 \mathrm{~s}$ (blue), and $1 \mathrm{~s} n \mathrm{p}$ (red) excited states. The solid arrow indicates the single-photon excitation of the $1 \mathrm{~s} 2 \mathrm{p}$ and $1 \mathrm{~s} n \mathrm{p}$ (red) states, whereas the dotted arrows indicate XUV absorption via two-color multi-photon excitation to $1 \mathrm{~s} n \mathrm{p}$ states with $n \geq 6$. Dashed lines indicate absorption lines corresponding to laser-induced virtual states. (b) Normalized photoabsorption cross-sections based on TDSE simulation of helium (see SI) when the attosecond XUV pulse overlaps in time with the peak of the NIR laser field. By removing the $1 \mathrm{~s} 2 \mathrm{~s}$ and $1 \mathrm{~s} 4 \mathrm{~s}$ states (blue) or 1s3s and $1 \mathrm{~s} 3 \mathrm{~d}$ states (green) from the calculation, we can confirm that the additional absorption lines are due to multi-photon excitation to those excited states.

number of NIR photons which must be absorbed or emitted. For example, the blue and green dashed lines in Fig. 2a are designated as $4 \mathrm{~s}^{-}$and $3 \mathrm{~d}^{-}$, respectively, while the red dashed line is designated as $n \mathrm{p}^{2-}(n \geq 6)$. The assignment of the excited states was confirmed by solution of the time-dependent Schrödinger equation (TDSE) in the two-color field, as shown in Fig. $2 \mathrm{~b}$ and discussed in more detail in the Supplementary Information (SI). Due to the broad bandwidth of the few-cycle laser field and the relative proximity of the excited states, the virtual state absorption structures overlap in many cases, and it is impossible to separate the contributions of several excited states and determine the final state unambiguously (for example, $3 \mathrm{~s}$ and $3 \mathrm{~d}^{-}$as well as $2 \mathrm{~s}^{+}$and $4 \mathrm{~s}^{-}$), as shown in Fig. $2 \mathrm{~b}$.

Unlike the single-XUV photon absorption cross section for excitation to the 1snp states in the absence of the dressing laser field, which does not depend on the sub-cycle field oscillations of the XUV pulse, the virtual state absorption features evolve dynamically with the delay, as the probability of exciting a virtual state depends intimately on the timing of the NIR laser field with respect to the attosecond pulse. Fig. 3a shows the absorbance spectrum as a function of the time delay for a dressing laser intensity of $1 \times 10^{13} \mathrm{~W} / \mathrm{cm}^{2}$. Negative delays indicate that the attosecond pulse arrives on the target before the NIR pulse. While the new virtual state absorption structures are most prominent near zero delay where the two pulses overlap, they can still be observed for large negative delays. Additionally, virtual state absorption features above the ionization threshold $\left(n \mathrm{~s}^{+}\right.$and $n \mathrm{~d}^{+}, 25-$ $28 \mathrm{eV})$ and below the $1 \mathrm{~s} 2 \mathrm{p}$ energy level $\left(2 \mathrm{~s}^{-}, \sim 19 \mathrm{eV}\right)$ are revealed in the time-dependent measurement. As the time delay is scanned with attosecond precision, the absorption strength of each feature is modulated on timescales faster than the laser cycle period. We find that the absorption strength in the vicinity of the $2 \mathrm{~s}^{+}, 3 \mathrm{~s}^{-}, 4 \mathrm{~s}^{-}$, and $3 \mathrm{~d}^{-}$ absorption features is modulated with a half-cycle periodicity, whereas the absorption strength near the $n \mathrm{p}^{2-}$ absorption feature oscillates with a quarter-cycle periodicity, as shown in Fig. $3 b$. Furthermore, the $1 \mathrm{~s} n \mathrm{p}$ absorption line energies change dynamically near zero delay, revealing Autler-Townes splitting of the 1s $2 p$ absorption line in addition to sub-cycle AC Stark and ponderomotive energy level shifts of the 1s3p and higher-lying states (see SI).

\section{Discussion}

Careful inspection of the data in Figure 3a, assisted by numerical simulations of the laser-dressed atom dynamics ${ }^{15,16}$, reveals two
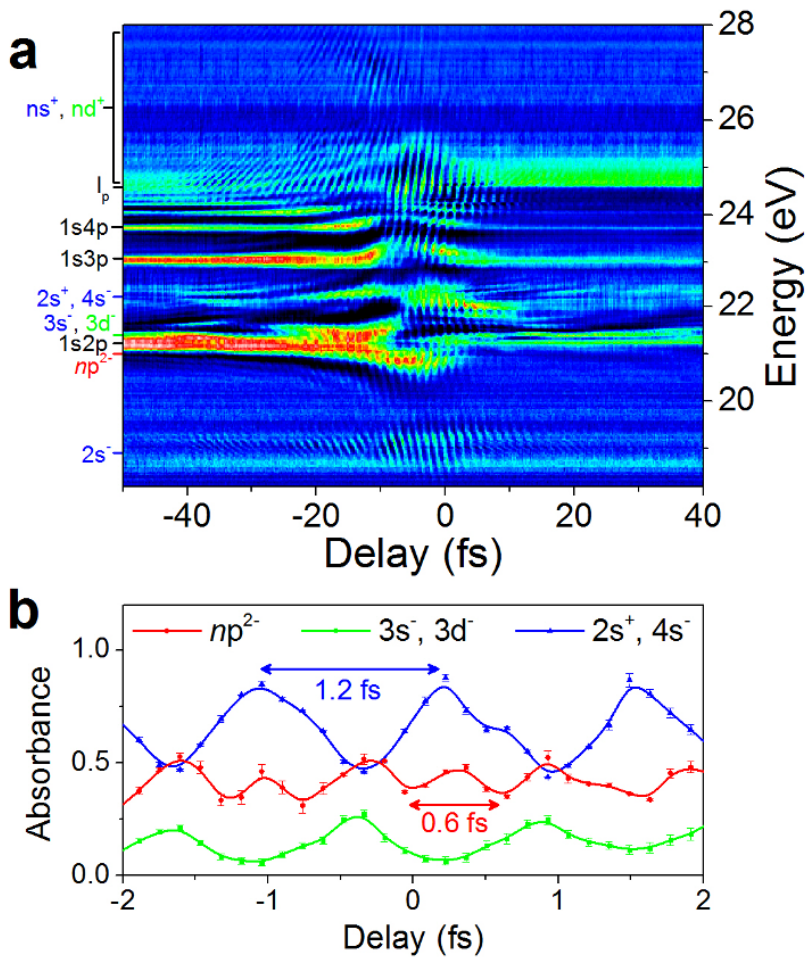

Figure $3 \mid$ Attosecond time-resolved absorption dynamics. (a) Timedelay-dependent absorbance spectrum with a dressing laser intensity of $10^{13} \mathrm{~W} / \mathrm{cm}^{2}$. With the strong dressing laser field, quantum-optical interferences can be observed in the absorption at negative delays. Near zero delay, the attosecond and NIR laser pulses overlap, and the absorption is dominated by the sub-cycle AC Stark and ponderomotive energy level shifts. (b) Absorbance lineouts in the vicinity of the transient virtual states. The absorbance (data points) was obtained by integrating the signal in the vicinity $( \pm 50 \mathrm{meV})$ of the virtual intermediate states indicated in Fig. 3a, and the error bars indicate the standard deviation of the absorbance in that region. The solid lines serve to guide the eye. While the absorbance near the $3 \mathrm{~s}^{-}, 3 \mathrm{~d}^{-}, 2 \mathrm{~s}^{+}$, and $4 \mathrm{~s}^{-}$virtual states oscillates with half-cycle periodicities, the absorbance near the $n \mathrm{p}^{2-}$ absorption feature oscillates with a quartercycle periodicity. 
distinct mechanisms by which the absorption features are modulated on the attosecond timescale. Figure 4a shows the calculated delaydependent absorption of an isolated attosecond pulse by the laserdressed helium atom, using laser pulse durations, central frequencies, and intensities identical to those used in the experiment (see Methods). Many of the dynamic features observed in the experiments can also be found in the simulation, including the absorption features corresponding to transient virtual states and the energy level dynamics of the $1 \mathrm{~s} 2 \mathrm{p}$ absorption line.

At negative delays, both the experiment and simulation reveal a relatively weak modulation of the absorption which appears primarily below $20 \mathrm{eV}$ and above the ionization threshold. These modulations follow hyperbolic lines of constant phase, which indicate two-path interference. Such interference was previously observed above the ionization threshold using photoelectron spectroscopy ${ }^{9}$, and was interpreted as the interference between electrons ionized directly by absorption of an XUV photon and indirectly by excitation of the $1 \mathrm{~s} 3 \mathrm{p}, 1 \mathrm{~s} 4 \mathrm{p}$, and $1 \mathrm{~s} 5 \mathrm{p}$ followed by absorption of an NIR photon. We can analyze the interference by taking the Fourier transform (FT) of Figures $3 \mathrm{a}$ and $4 \mathrm{a}$ along the delay axis, as shown in Fig. 4b. The FT spectrograms for the negative delay regions $\left(-40 f_{s}<\tau_{D}<-20 f_{s}\right)$ are shown for the experimental data in the left panel and for the simulation in the right panel. In both cases, the absorption structures appear as $45^{\circ}$ lines with Fourier frequencies equal to $\omega-E_{1 s n p}$, where $\omega$ is the XUV photon energy and $E_{1 s n p}$ is the 1snp excited state energy. The dominant interference is observed for $E_{1 s 2 p}=21.22 \mathrm{eV}$, although interferences with $E_{1 s 3 p}=23.09 \mathrm{eV}$ can also be observed in the simulated data, as indicated by the dashed lines in Fig. 4b. The two-path interference can be seen extending to photon energies of $28 \mathrm{eV}$ owing to the high spectral resolution of the XUV spectrometer.

The interferences in the attosecond transient absorption measurement, however, cannot be explained using the electron interference model. This is obvious for two reasons: (1) whereas the photoelectron measurement can only measure interferences for electrons arriving on the detector with the same final energy, the attosecond transient absorption measurement can only measure interferences of quantum pathways for which the initial XUV photon energy is the same; and (2) no "direct" pathway exists for exciting an electron with a photon energy below $20 \mathrm{eV}$, since no bound states exist with energy levels below $E_{1 s 2 s}=20.61 \mathrm{eV}$. Instead, the observed interference should be described as quantum optical interferences. The absorption of light by the atom can be described by the Beer-Lambert law ${ }^{15,16}$ :

$$
\left|\tilde{\varepsilon}_{\text {out }}(\omega)\right|^{2}=\left|\tilde{\varepsilon}_{\text {in }}(\omega)\right|^{2} \exp \left\{-\frac{2 \pi \omega}{c} \Im\left[\frac{\tilde{P}(\omega)}{\tilde{\varepsilon}_{\text {in }}(\omega)}\right] N L\right\},
$$

where $\tilde{\varepsilon}_{\text {in }}(\omega)$ and $\tilde{\varepsilon}_{\text {out }}(\omega)$ are the XUV spectrum amplitudes before and after interaction with the atom, respectively, $N L$ is the densitylength product, and $\tilde{P}(\omega)$ is the Fourier transform of the timedependent polarization $\langle\Psi(\mathbf{r}, t)|z| \Psi(\mathbf{r}, t)\rangle$ of the atom with the wavefunction $\Psi(\mathbf{r}, t)$.

In the absence of the laser, the output spectrum far from any resonances is equal to the input spectrum, and therefore arises from a frequency-dependent polarization with no imaginary component, $\Im[\tilde{P}(\omega)]=0$. This implies that the phase of the polarization, $\arg [\tilde{P}(\omega)]$, is also zero. However, the dressing laser can also excite polarization with frequencies far from the $1 \mathrm{~s} n \mathrm{p}$ resonances due to the formation of transient excited states. Since the population of the transient states, and therefore the instigation of the frequency-shifted polarization, is initiated by the arrival of the dressing laser pulse, a phase difference of $\left(\omega-E_{1 s n p}\right) \tau_{D}$ arises between the two components of the frequency-dependent polarization. The interference fringes will therefore follow the hyperbolic lines of constant phase, becoming more closely spaced in energy for larger negative time delays, and the absorption features will oscillate on the attosecond timescale.

Near zero delay, however, the absorption modulations are no longer consistent with the two-path interference. In this region, the periodicity of the oscillation does not change with the XUV photon
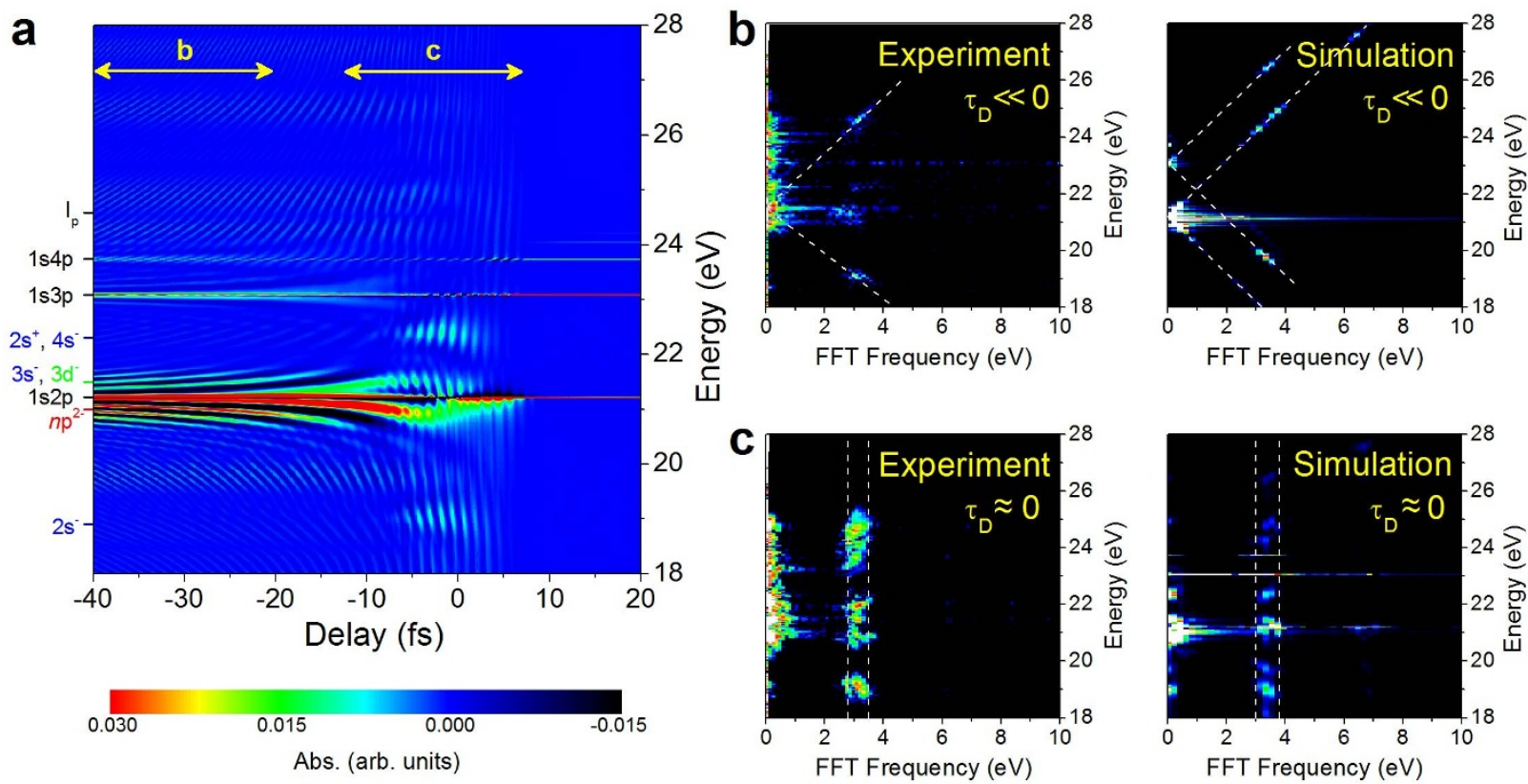

Figure $4 \mid$ Quantum-optical interferences and sub-cycle energy level shifts. (a) Simulated time-delay-dependent absorbance spectrum with a dressing laser intensity of $10^{13} \mathrm{~W} / \mathrm{cm}^{2}$. The spectrogram can be divided into two regions to separate the effects of quantum-optical interferences and sub-cycle energy level dynamics. (b) Fourier transform analysis of region b, which spans $-40 f_{s}<\tau_{D}<-20 f_{s}$. The FT spectrogram obtained from the experimental data in Fig. 3a is shown in the left panel, while the right panel shows that obtained from the simulated data in Fig. 4a. The dashed 45-degree lines indicate the two-path quantum-optical interference. (c) Fourier transform analysis of region c, which spans $-13 f_{\mathcal{S}}<\tau_{D}<7 f_{s}$. Half- and quarter-cycle oscillations are evident above and below the ionization potential, and the oscillation frequency no longer follows the 45-degree lines associated with the two-path interference. 
energy, as shown in the FT spectrograms of Fig. 4c, indicating that it is not a result of the direct-indirect interference. In this case, the XUV pulse does not see the field-free atom, and the laser-induced couplings occur simultaneously with the population of the excited states. Therefore, we must instead consider the XUV absorption of the laser-perturbed atom. In the simulations, we find that the half- and quarter-cycle oscillations in the transient excited state absorption features are evident in the Fourier transform spectrogram, resulting from the two-color multi-photon absorption.

On the other hand, the absorption dynamics near the ionization threshold are dominated by sub-cycle AC Stark and ponderomotive energy level shifts, which result from the strong laser-induced bound-bound and bound-continuum state couplings. Since the simulations neglect bound-continuum state couplings, the AC Stark and ponderomotive shifts cannot be reproduced well in the simulation, and the oscillation amplitudes in the simulated FT spectrogram near the ionization threshold are smaller than those in the experiment. However, we do observe AC Stark energy level shifts and absorption threshold shifts corresponding to the ponderomotive shift of the ionization potential in the experimental data, which exhibit a strong half-cycle periodicity (see SI). The sub-cycle ponderomotive shift follows the square of the instantaneous laser field strength, analogous to the previously-observed sub-cycle AC Stark shifts $^{6}$. However, whereas the measurement of the sub-cycle AC Stark shifts by transient absorption is necessarily convoluted due to the relatively long lifetimes of the laser-dressed excited states ${ }^{6,15}$ resulting in a relatively small modulation depth, the sub-cycle ponderomotive shift can be observed more clearly, as it relies on continuum states without finite ionization lifetimes.

The absorption of light is a fundamental process which can be used to initiate chemical reactions ${ }^{17}$ or as a basis for lightwave electron$\mathrm{ics}^{18}$. Here, we show that a moderately intense $\left(10^{11}-10^{13} \mathrm{~W} / \mathrm{cm}^{2}\right)$ laser field can control the absorption of light over a broad photon energy range on the attosecond timescale. When overlapped in space and time with a few-cycle laser field, new features in the absorption spectrum of an isolated attosecond pulse appear, corresponding to virtual intermediate states in the two- and three-photon excitation to bound states which cannot be populated by the XUV pulse alone. These absorption features can be "gated" on the half- and even quarter-cycle timescale by changing the delay between the two pulses, allowing for all-optical switching on the attosecond timescale.

\section{Methods}

Isolated attosecond pulses were produced from $5 f s$ near-infrared driving pulses centered at $730 \mathrm{~nm}$ using the generalized double optical gating (GDOG) ${ }^{19}$ technique in argon gas or the ionization gating ${ }^{20}$ technique in xenon gas. The generalized double optical gating optics consist of a quartz waveplate with optical axis set at $45^{\circ}$ relative to the laser polarization axis, two glass plates at the Brewster angle, a second quartz waveplate with optical axis set along the laser polarization axis, and a second harmonic generating crystal. For the ionization gating, the optical axis of the first quartz plate was instead set along the laser polarization axis, and the glass plates were set at normal incidence. In this configuration, no second harmonic light is generated and the incident laser is linearly polarized. The isolated attosecond pulse was then recombined with the few-cycle laser field using a Mach-Zehnder interferometer with active stabilization of the delay ${ }^{7}$. The intensity of the NIR laser was controlled using an iris. Both pulses were then focused to the second gas cell (1.5 $\mathrm{mm}$ inner diameter), which was backed with 40 Torr of helium gas to absorb $\sim 75 \%$ of the spectrum near $25 \mathrm{eV}$. Absorption near the $1 \mathrm{~s} n \mathrm{p}$ excited state energy levels became comparable to the above-threshold absorption with the addition of the laser field. The transmitted spectra were measured using a home-built XUV spectrometer ${ }^{21}$ based on a flat-field grazing incidence reflection grating (Hitachi 001-0640) as a function of the time delay, with delay steps of 140 as. The spectrometer resolution was approximately $50 \mathrm{meV}$ within the energy range of interest.

The numerical simulations shown in Fig. $2 \mathrm{~b}$ and Fig. $4 \mathrm{a}$ were carried out following the formalisms developed by Santra et al..$^{15}$ and by Pfeiffer and Leone ${ }^{16}$ for calculation of the transient absorption of reduced dimensionality systems. The laser-dressed atom wavefunction $\Psi(\mathbf{r}, t)$ was expanded on the basis set of the field-free atom $\psi_{1 \text { snl }}(\mathbf{r})$ as:

$$
|\Psi(\mathbf{r}, t)\rangle=\sum_{n, l} c_{1 s n l}(t)\left|\psi_{1 s n l}(t)\right\rangle,
$$

and the time-dependent state amplitudes $c(t)$ were obtained by numerically solving the coupled differential equations (in atomic units):

$$
i \ddot{c}_{a}=E_{a} c_{a}(t)-\sum_{k \neq a}\left\langle\psi_{k}(\mathbf{r})|z| \psi_{a}(\mathbf{r})\right\rangle F(t) c_{k}(t)
$$

using tabulated values for the excited state energies $E_{a}$ and the dipole matrix elements $\left\langle\psi_{k}(\mathbf{r})|z| \psi_{a}(\mathbf{r})\right\rangle^{10}$ with a fourth-order Runge Kutta differential equation solver. Here $k$ and $a$ represent individual states specified by the quantum numbers $n$ and $l$, and $F(t)$ represents the combined XUV and NIR laser fields. States with $n$ ranging from 1 to 8 and with $l=s, p$, and $d$ were included in the calculation. Additional TDSE simulations based on the non-perturbative treatment of the NIR field by means of non-Hermitian Floquet theory ${ }^{22}$ and first-order perturbation theory with respect to the attosecond pulse were used to confirm the reduced-dimensionality simulations. The details of these calculations can be found the SI.

1. Fano, U. \& Cooper, J. W. Spectral Distribution of Atomic Oscillator Strengths. Rev. Mod. Phys. 40, 441 (1968).

2. Loh, Z.-H., Greene, C. H. \& Leone, S. R. Femtosecond induced transparency and absorption in the extreme ultraviolet by coherent coupling of the He $2 \mathrm{~s} 2 \mathrm{p}\left({ }^{1} \mathrm{P}^{\circ}\right)$ and $2 \mathrm{p}^{2}\left({ }^{1} \mathrm{~S}^{\mathrm{e}}\right)$ double excitation states with $800 \mathrm{~nm}$ light. Chem. Phys. 350, 7 (2008).

3. Glover, T. E. et al. Controlling X-rays with light. Nature Physics 6, 69 (2010).

4. Gouleilmakis, E. et al. Real-time observation of valence electron motion. Nature 466, 739 (2010).

5. Wang, H. et al. Attosecond Time-Resolved Autoionization of Argon. Phys. Rev. Lett. 105, 143002 (2010).

6. Chini, M. et al. Subcycle ac Stark shift of Helium Excited States Probed with Isolated Attosecond Pulses. Phys. Rev. Lett. 109, 073601 (2012).

7. Chini, M. et al. Delay control in attosecond pump-probe experiments. Opt. Exp. 17, 21459 (2009).

8. Loh, Z.-H., Khalil, M., Correa, R. E. \& Leone, S. R. A tabletop femtosecond timeresolved soft x-ray transient absorption spectrometer. Rev. Sci. Instrum. 79, 073101 (2008).

9. Mauritsson, J. et al. Attosecond Electron Spectroscopy Using a Novel Interferometric Pump-Probe Technique. Phys. Rev. Lett. 105, 053001 (2010).

10. Drake, G. W. F. High Precision Calculations for Helium. in Springer Handbook of Atomic, Molecular and Optical Physics, edited by Drake, G. W. F. (Springer, New York 2006).

11. Heron, S., McWhirter, R. W. P. \& Rhoderick, E. H. Measurements of Lifetimes of Excited States of Helium Atoms. Proc. Roy. Soc. London Ser. A Math. Phys. Eng. Sci. 234, 565 (1956).

12. Tong, X. M. \& Toshima, N. Controlling atomic structures and photoabsorption processes by an infrared laser. Phys. Rev. A 81, 063403 (2010).

13. Gaarde, M. B., Buth, C., Tate, J. L. \& Schafer, K. J. Transient absorption and reshaping of ultrafast XUV light by laser-dressed helium. Phys. Rev. A 83, 013419 (2011).

14. Chen, S., Schafer, K. J. \& Gaarde, M. B. Transient absorption of attosecond pulse trains by laser-dressed helium. Opt. Lett. 37, 2211 (2012).

15. Santra, R., Yakovlev, V. S., Pfeifer, T. \& Loh, Z.-H. Theory of attosecond transient absorption spectroscopy of strong-field generated ions. Phys. Rev. A 83, 033405 (2011).

16. Pfeiffer, A. N. \& Leone, S. R. Transmission of an isolated attosecond pulse in a strong-field dressed atom. Phys. Rev. A 85, 053422 (2012).

17. Kling, M. F. \& Vrakking, M. J. J. Attosecond electron dynamics. Annu. Rev. Phys. Chem. 59, 463 (2008).

18. Goulielmakis, E. et al. Attosecond Control and Measurement: Lightwave Electronics. Science 317, 769 (2007).

19. Feng, X. et al. Generation of Isolated Attosecond Pulses with 20 to 28 Femtosecond Lasers. Phys. Rev. Lett. 103, 183901 (2009).

20. Ferrari, F. et al. High-energy isolated attosecond pulses generated by abovesaturation few-cycle fields. Nature Photonics 4, 875 (2010).

21. Wang, X. et al. In situ calibration of an extreme ultraviolet spectrometer for attosecond transient absorption experiments. Applied Optics 52, 323 (2013).

22. Chu, S. I. \& Telnov, D. A. Beyond the Floquet theorem: generalized Floquet formalisms and quasienergy methods for atomic and molecular multiphoton processes in intense laser fields. Phys. Rep. 390, 1 (2004).

\section{Acknowledgements}

This work is supported by the U. S. Army Research Office and by the National Science Foundation. D. Z., D. A. T., and S. I. C. acknowledge partial support from the U. S. Department of Energy. S. I. C. would like to recognize also the partial support from National Taiwan University.

\section{Author contributions}

Z. C. and M. C. conceived the experiment. M. C., X. W., Y. C., and Y. W. developed the attosecond transient absorption setup and carried out the experiments. M. C. and X. W. analyzed the data. M.C., D. Z., D. A. T., and S. I. C. performed the numerical simulations M. C., X. W., D. Z., D. A. T., S. I. C., and Z. C. wrote the manuscript. 


\section{Additional information}

Supplementary information accompanies this paper at http://www.nature.com/ scientificreports

Competing financial interests: The authors declare no competing financial interests. License: This work is licensed under a Creative Commons
Attribution-NonCommercial-NoDerivs 3.0 Unported License. To view a copy of this license, visit http://creativecommons.org/licenses/by-nc-nd/3.0/

How to cite this article: Chini, M. et al. Sub-cycle Oscillations in Virtual States Brought to Light. Sci. Rep. 3, 1105; DOI:10.1038/srep01105 (2013). 